\title{
A 1 Gbps 105.4 GHz Link with a Directly Modulated Photonic Integrated Dual Laser Source
}

\author{
Z. Yang ${ }^{1}$, M. J. Fice ${ }^{2}$, K. Balakier ${ }^{2}$, A. Wonfor ${ }^{1}$, C. Renaud $^{2}$, G. Carpintero ${ }^{3}$, G.Kervella ${ }^{4}$, F. van Dijk ${ }^{4}$, \\ A. J. Seeds ${ }^{2}$, R. V. Penty ${ }^{1}$, I. H. White ${ }^{1}$ \\ ${ }^{I}$ Electrical Division, Department of Engineering, University of Cambridge, 9 JJ Thomson Avenue, Cambridge CB3 0FA, UK, zy235@cam.ac.uk \\ ${ }^{2}$ Department of Electronic and Electrical Engineering, University College London, Torrington Place, London, WC1E 7JE, UK \\ ${ }^{3}$ Universidad Carlos III de Madrid, Av de la Universidad, 30. Leganés 28911 Madrid, Spain \\ ${ }^{4}$ III-V Lab, a joint lab of Thales Research and Technology, Alcatel-Lucent Bell Labs France, and CEA Leti, 91767 Palaiseau Cedex, France
}

Abstract: A 1 Gbps $105.4 \mathrm{GHz}$ wireless link is demonstrated by directly modulating a photonic integrated duallaser source. A $50 \mathrm{~m}$ link is predicted to be able to achieve error free operation using FEC following optimisation. OCIS codes: (060.5625) RF photonics; (130.3120) Integrated optics devices.

\section{Introduction}

The increasing data rates required for wireless data communications, along with the need for short distance point-topoint wireless connections, are stimulating research in wireless links with carrier frequencies in the sub-terahertz range [1]. Such links will require compact and low-cost sub-THz transmitters operating at gigabits per second data rates. Furthermore, the carrier frequencies of the links should be widely tuneable if multi-channel communications is to be provided. These requirements, however, are challenging for all-electronic approaches [2] and terahertzquantum-cascade-lasers [3]. Resonant tunnelling diodes have been reported with a data rate of $2 \mathrm{Gbps}$ at $542 \mathrm{GHz}$ within a back-to-back connection, though the carrier frequency of this particular sub-THz source however has a limited tuning range [2]..

As a result, in recent years, research interest has focused on using photonic techniques. For example, studies have been reported into an integrated tunable sub-THz transmitter [4,5] comprising two distributed feedback (DFB) lasers and a passive combiner. A sub-THz signal is generated by detecting the combined optical signals from the lasers using a high bandwidth photodiode connected to a horn antenna. The signal frequency is set by the separation of the laser wavelengths, which enables wide frequency tuneability of such devices of up to $120 \mathrm{GHz}$ being reported [4]. A link based on a dual laser source has been demonstrated by applying $1 \mathrm{Gbps}$ on-off-keying (OOK) data onto a Mach-Zehnder modulator [5]. In that case, however, the photonic link components were not fully integrated.

In this paper, we demonstrate a transmission link consisting of a compact dual-laser optical source with an onchip modulator. The optical source generates a beat frequency of $105.4 \mathrm{GHz}$ which is then transmitted into free space. 1 Gbps OOK data with a run length of $2^{7}-1$, similar to the block code run length of datacoms standards such as $\mathrm{GbE}$, is directly applied to the on-chip modulator. A chirp-enhanced direct modulation scheme [6] is employed to enhance the received signal extinction ratio. The resultant signal captured is evaluated to have a bit error rate (BER) of $10^{-3}$, the forward error correction (FEC) error rate limit, at a transmission length of $40 \mathrm{~cm}$. The achieveable link length can be predicted to be $50 \mathrm{~m}$ at $1 \mathrm{Gbps}$ with optimised link antenna and RF amplifier gains and minimised waveguide losses.

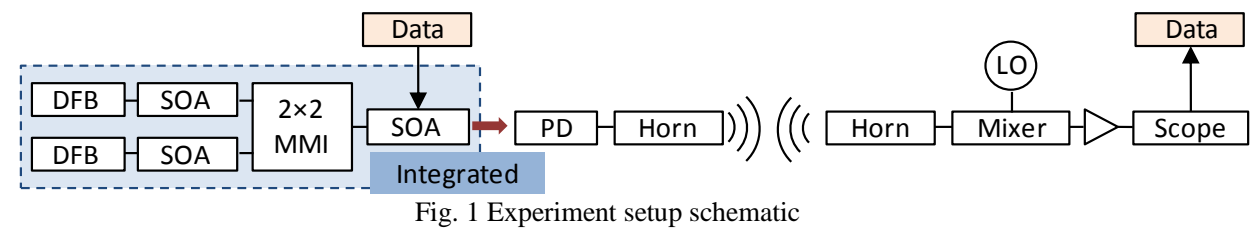

\section{Experiment setup and results}

As shown in the blue block of Fig. 1, the photonic integrated device incorporates two DFB lasers and two semiconductor optical amplifiers (SOAs) which couple to a $2 \times 2$ multimode interference (MMI) coupler. An SOA is integrated at the MMI's output port, not only to provide additional gain but also to provide modulation. The modulator-SOA modulation bandwidth is limited by the sub-mount de connection with a bandwidth of $500 \mathrm{MHz}$. An optical tone is generated by combining the outputs of the DFBs through the MMI, using a lensed fibre coupled power of up to $4 \mathrm{~mW}$. The photonic components on the chip are biased at $95 \mathrm{~mA}$ (DFB1), $240 \mathrm{~mA}$ (DFB2), $51 \mathrm{~mA}$ (SOA1), $40 \mathrm{~mA}$ (SOA2) and $60 \mathrm{~mA}$ (modulator), respectively, to generate a beat frequency of $105.4 \mathrm{GHz}$. The modulator-SOA is directly modulated by a 1 Gbps $2^{7}-1$ run length PRBS with a modulation current of $7 \mathrm{~mA}$. The resultant optical output exhibits frequency chirp to be used by a chirp-enhanced modulation technique [6]. For 
transmission studies, the optical output is connected to a photodiode (PD) with a $-6 \mathrm{dBe}$ bandwidth of $100 \mathrm{GHz}$ and a dc responsivity of $0.48 \mathrm{~A} / \mathrm{W}$. The output of the photodiode is connected to a $90-140 \mathrm{GHz}$ band horn antenna via waveguides (W1-W1, W1-WR10 and WR10-WR8). The gain of the transmitter and receiver horn antennas are 23 $\mathrm{dBi}$ and $20 \mathrm{dBi}$ respectively. The RF signal at the receiver horn antenna is down-converted to an IF signal by using a 90-140 GHz Virginia Diodes sub-harmonic mixer with a conversion loss of -6 to $-10 \mathrm{~dB}$ and a $51.3 \mathrm{GHz}$ local oscillator. As shown in Fig. 2(a), the IF signal has a frequency of $2.8 \mathrm{GHz}$. The IF signal is then amplified (20 dB) and captured by a $3.5 \mathrm{GHz}$ bandwidth real time scope with a $10 \mathrm{Gs} / \mathrm{s}$ sampling rate and a real time window of 0.1 $\mathrm{ms}$. The captured signals, consisting of $10^{5}$ bits, can be offline processed and compared with the PRBS sequence to evaluate the BER performance. A bandpass filter is applied in the offline process to enhance the logical ones to zeros extinction ratio by locating the filter central frequency with the peak frequency of the ones. The filtered signal can be demodulated by using an envelope detection process. The resulting eye diagram is shown in Fig. 2(b).

To evaluate the signal BER performance, the transmission length and the optical power to the PD are varied, respectively. In Fig. 2(c) the distance between the two horn antennas is varied from 20 to $80 \mathrm{~cm}$ causing a change in the received RF power. The RF power is calculated by taking the mixer conversion loss, the RF amplifier gain and the captured IF signal power into account. Similarly, the RF power can effectively be varied by the PD optical input. The results agree well and show that $-44 \mathrm{dBm}$ RF power is required using FEC (pre-FEC BER $=10^{-3}$ ).
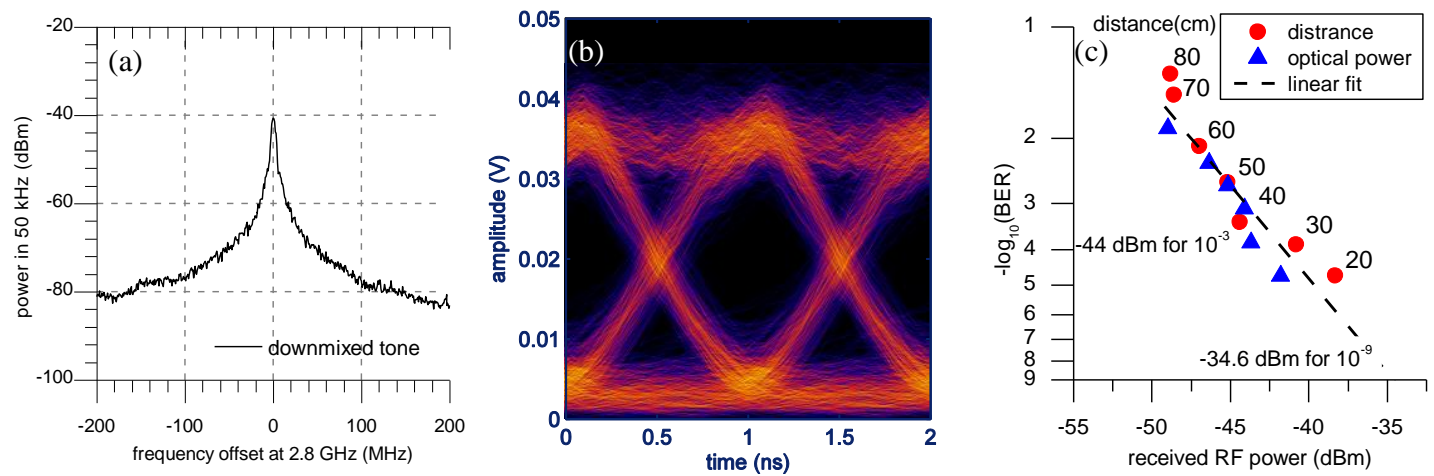

Fig. 2 Experiment results: (a) down-converted RF tone at the receiver; (b) 1 Gbps eye diagram generated by using the real-time captured signal; (c) BER performance versus the received RF signal power. The red dots are measured by varying the transmission distance, while the blue dots by varying the optical power injected into the PD.

An improved power budget for the link can be calculated as follows. The loss of the waveguides between the PD and the transmitter horn is $-6.6 \mathrm{~dB}$. The free space propagation loss is measured and in line with the free space path loss model. The loss of the waveguides can be avoided by enabling direct connection between the PD and the horn. The receiver horn gain can be increased to $23 \mathrm{dBi}$ (ie the same as the transmitter). The receiver RF amplifier gain can be increased to $30 \mathrm{~dB}$. Therefore, with the optimised link power budget, the link loss can be reduced by $19.6 \mathrm{~dB}$. With an optical power input to the PD of $12.6 \mathrm{dBm}$, the link length is predicted to extend to $50 \mathrm{~m}$ for a pre-FEC BER of $10^{-3}$.

\section{Conclusion}

We present a photonic integrated dual laser source for a $120 \mathrm{GHz}$ band link with a data rate of $1 \mathrm{Gbps}$. The source is directly modulated with a $2^{7}-1$ run length OOK PRBS. At the receiver, the down-converted real time signal is offline processed by simulating a bandpass filter to select the frequency chirped logical ones and an envelope detector. An error free link within a FEC limit is demonstrated over a $40 \mathrm{~cm}$ link. This work has the potential of providing a high speed, low cost and compact solution for $50 \mathrm{~m}$ links with optimised connections.

\section{References}

[1] A. Stohr, "Pushing the Boundaries", Microwave Magazine, no. June, p. 106, Jan. 2009.

[2] K. Ishigaki et al, "Direct Intensity Modulation and Wireless Data Transmission Characteristics of Terahertz-oscillating Resonant Tunnelling Diodes", Electronics Letters, vol. 48, no. 10, p. 582, 2012.

[3] P. H. Siegel, "Terahertz Technology", 2002.

[4] F. van Dijk et al, "Monolithic dual wavelength DFB lasers for narrow linewidth heterodyne beat-note generation", 2011 MWP, pp. 73-76, Oct. 2011.

[5] M. Fice et al, "146-GHz Millimeter-wave Radio-over-fiber Photonic Wireless Transmission System”, Optics Express, vol.20, no. 2, p. 1769, 2012.

[6] Z. Yang et al, "Chirp-Enhanced Direct Modulation of a Monolithic Sub-Terahertz Dual Laser Transmitter”, MWP, 2013 